\title{
Efeito do estresse sobre o metabolismo glicídico de camundongos tornados obesos
}

\author{
Effect of stress on glucose metabolism in obese-induced mice
}

Gabriela Fernandes Gozoli ${ }^{1}$, Paula Regina Manuner Zeca ${ }^{1}$, Bruna Calzzetta ${ }^{1}$, Mário Luís Ribeiro Cesaretti ${ }^{1}$

\begin{abstract}
RESUMO
Introdução: Distúrbios do sono, isoladamente ou em associação a uma dieta rica em calorias, podem determinar alterações metabólicas no sistema nervoso autônomo e no eixo hipotálamo-hipófise-adrenal, os quais podem aumentar a glicemia e produzir intolerância à glicose. $\mathrm{O}$ estado de intolerância à glicose geralmente precede o aparecimento de diabetes tipo 2. Objetivo: Este estudo visou avaliar os efeitos da associação da privação de sono paradoxal e dieta hipercalórica sobre o comportamento alimentar e a tolerância à glicose de camundongos. Métodos: Camundongos Swiss foram distribuídos em seis grupos: (1) controle; (2) dieta hipercalórica; (3) privação de sono paradoxal; (4) privação de sono paradoxal + dieta hipercalórica; (5) monoglutamato de sódio (MSG); e (6) privação de sono paradoxal + monoglutamato de sódio (MSG+PSP). Durante as oito semanas de acompanhamento, o consumo alimentar foi avaliado e os camundongos pesados periodicamente. A privação de sono foi realizada semanalmente. Após oito semanas executou-se o teste de tolerância oral à glicose (TTGO) e de sensibilidade à insulina (TTI). Ao final, houve pesagem do coração e da gordura epididimal. Resultados: Houve aumento significante do peso corporal nos camundongos tornados obesos, que se associou com o avanço do conteúdo de gordura visceral. Todos os animais, obesos e com privação de sono paradoxal, tiveram piora no metabolismo de glicose medido pelo TTGO e TTI. Não houve mudança no peso cardíaco. Conclusão: A administração de uma dieta hipercalórica e a privação de sono paradoxal determinam intolerância à glicose e resistência à insulina, que nesse trabalho não foi sinérgico.
\end{abstract}

Palavras-chave: sono REM; obesidade; transtornos do metabolismo de glucose; camundongos.

\begin{abstract}
Introduction: Sleep disorders, alone or in association with a high-calorie diet, may determine metabolic changes in the autonomic nervous system and in the hypothalamic-pituitary-adrenal axis, which may increase glycemia and produce glucose intolerance. The state of glucose intolerance usually precedes the onset of type 2 diabetes. Objective: This study aims to evaluate the effects of the association of paradoxical sleep deprivation and hypercaloric diet on the eating behavior and glucose tolerance of mice. Methods: Swiss mice were distributed into six groups: (1) control; (2) hypercaloric diet; (3) paradoxical sleep deprivation; (4) paradoxical sleep deprivation + hypercaloric diet; (5) monossodium glutamate (PSP); and (6) paradoxical sleep deprivation + monossodium glutamate. During the eight-week follow-up, food consumption was assessed and mice were weighed periodically. Sleep deprivation was performed weekly. After eight weeks, the oral glucose tolerance (TTGO) and insulin sensitivity test (TTI) were performed. At the end, heart and epididymal fat were weighed. Results: There was a significant increase in body weight in obese mice, which was associated with increased visceral fat content. All animals, obese and PSP, showed worsening in the parameters that measured the glucose metabolism, either on TTGO and TTI. There was no change in cardiac weight. Conclusion: The administration of a hypercaloric diet and paradoxical sleep deprivation determine glucose intolerance and insulin resistance, which in this work was not synergistic. Keywords: sleep, REM; obesity; glucose metabolism disorders; mice.
\end{abstract}

${ }^{1}$ Pontifícia Universidade Católica de São Paulo (PUC-SP), Faculdade de Ciências Médicas e da Saúde (FCMS) Sorocaba (SP), Brasil.

Contato: mcesaretti@ pucsp.br

Recebido em 26/09/2016. Aceito para publicação em 09/05/2017. 


\section{INTRODUÇÃO}

$\mathrm{O}$ estresse do mundo contemporâneo está associado a diversas mudanças no estilo de vida do homem moderno. Entre essas mudanças estão o menor número de horas dormidas. ${ }^{1}$ Estudos recentes mostram que o americano na década de 1960 dormia aproximadamente 8,0 a 8,9 horas, enquanto em 2004 esse tempo reduzia para somente 6 horas, sendo que $30 \%$ desses pacientes referiam dormir menos de 6 horas por dia., ${ }^{2,3}$ Ressalta-se que este estudo se refere apenas ao menor número de horas dormidas e não à qualidade do sono dos pacientes. Outros estudos referem que indivíduos com short-sleep ( $<7 \mathrm{~h} /$ noite) possuem aumento de mortalidade e morbidade para doenças cardiovasculares quando comparados com indivíduos long-sleep ( $>8 \mathrm{~h} /$ noite). ${ }^{4,5}$

Destaca-se que, associada a menor quantidade de horas de sono, os países desenvolvidos ${ }^{6}$ e o Brasil $^{7}$ vivem um aumento do número de pessoas com sobrepeso e obesidade, causando a diminuição da qualidade do sono dos indivíduos, por induzir ao ronco e à síndrome de apneia de sono (SAS) ${ }^{8,9}$ Sendo esse aumento de peso corporal associado à menor quantidade de atividade física e à ingesta de produtos com grande quantidade de calorias. ${ }^{10}$

Vários são os estudos epidemiológicos que associam o menor número de horas de sono com o aparecimento de diabetes mellitus tipo 2, como o Nurses Health Study, ${ }^{11}$ que mostrou aumento de incidência de diabetes mellitus tipo 2 em enfermeiras que dormiam aproximadamente cinco horas por noite. Em um estudo sueco, ${ }^{12}$ verificou-se que homens com idade entre 35 e 51 anos com dificuldade para dormir, ou ainda aqueles que faziam uso de benzodiazepínicos para esse fim, tiveram aumento da mortalidade para doenças cardiovasculares. Outro estudo mostra que a diminuição do sono para menos de $6 \mathrm{~h}$ /noite dobra o risco para o desenvolvimento de diabetes mellitus tipo $2 .^{13}$

A menor quantidade e/ou qualidade do sono poderia refletir em alterações do metabolismo da glicose, levando à intolerância à glicose e resistência à insulina. Também poderia induzir alterações do comportamento alimentar e menor gasto energético. Todos esses fatores poderiam culminar com o aparecimento de diabetes mellitus tipo $2 .{ }^{14}$

Essa alteração metabólica decorrente da menor quantidade e/ou qualidade do sono poderia direta ou indiretamente determinar a ativação do sistema nervoso autônomo simpático, aumentar a secreção de cortisol e diminuir o conteúdo de tireotropina. ${ }^{15}$

As elevações plasmáticas de insulina causam aumento dose-dependente de catecolaminas plasmáticas, levando ao aumento do débito cardíaco e produzindo vasoconstrição.

Outros estudos também demonstram que o aumento das concentrações plasmáticas de insulina determina elevação da frequência de disparos do nervo peroneal, demonstrando hiperatividade adrenérgica em fibras musculares. ${ }^{16}$ Por outro lado, a insulina determina aumento da velocidade do metabolismo da noradrenalina, porém, essa resposta está suprimida em indivíduos obesos e insulino-resistentes. ${ }^{17}$

O estudo de Spiegel et al., em 1999, demonstra bem a associação de diminuição de horas de sono com alterações me- tabólicas. Indivíduos saudáveis foram submetidos a um déficit de sono, os quais durante 6 dias permaneceram apenas 4 horas na cama. Esse período foi comparado com outro, no qual os indivíduos permaneceram 12 horas na cama. Verificou-se que no período de déficit de sono os pacientes tiveram:

- diminuição do metabolismo cerebral de glicose;

- $\quad$ aumento da atividade do sistema nervoso simpático;

- $\quad$ aumento das concentrações de cortisol;

- $\quad$ diminuição da captação periférica de insulina;

- $\quad$ diminuição da sensibilidade à insulina;

- $\quad$ além do aumento da produção de citocinas inflamatórias. ${ }^{18}$

Outra possível alteração metabólica ocorre no balanço entre peptídeos orexígenos e anorexígenos no centro de regulação do apetite (hipotálamo lateral e núcleo arqueado). ${ }^{19}$ Ratos que foram privados de sono paradoxal apresentaram hiperfagia associada ao aumento de orexinas A e B, aumento da secreção de neuropeptídeo Y, diminuição da leptina e aumento da secreção de grelina. ${ }^{20} \mathrm{O}$ mesmo resultado se repetiu em seres humanos que permaneceram na cama durante apenas 4 horas por 2 noites, seguido de outro período de 10 horas na cama por 2 noites. Nos indivíduos com menor número de horas dormidas, verifica-se diminuição da leptina (um hormônio anorexígeno), aumento da grelina (um hormônio orexígeno), mais fome, maior apetite, principalmente por carboidratos. ${ }^{1}$

O objetivo deste trabalho foi avaliar o efeito do estresse (privação de sono paradoxal) sobre o comportamento alimentar e metabolismo glicídico de camundongos submetidos a uma dieta hiperlipídica ou tornados obesos pela administração neonatal de monoglutamato de sódio.

\section{MATERIAL E MÉTODOS}

Para este estudo foram utilizados camundongos da cepa Swiss, machos, entre 30 e 35 gramas, cedidos pelo Biotério Central da Faculdade de Ciências Médicas e da Saúde da Pontifícia Universidade Católica de São Paulo (FCMS/PUC-SP). Os animais foram mantidos em condições ideais de biotério. Este trabalho foi aprovado pela Comissão de Ética Animal da FCMS/PUC-SP. O período experimental de acompanhamento foi de oito semanas.

\section{Grupos experimentais}

Os camundongos foram distribuídos nos seguintes grupos experimentais:

1. Grupo Controle (CONT; $n=6)$ : permaneceram em suas caixas durante todas as 8 semanas do estudo. Esses animais receberam dieta padrão para camundongos;

2. Grupo Dieta Hipercalórica (DHC; $n=6$ ): permaneceram em suas caixas durante todas as 8 semanas do estudo. Após o desmame ( $21^{\circ}$ dia), a dieta padrão foi trocada por uma hipercalórica (descrita a seguir);

3. Grupo Privação de Sono Paradoxal (PSP; $n=6$ ): foram submetidos semanalmente a uma sessão de privação de sono paradoxal de 48 horas (descrita a seguir). Esses animais receberam dieta padrão para roedores; 
4. Grupo Privação de Sono Paradoxal + Dieta Hipercalórica (DHC+PSP; $n=6$ ): foram submetidos a sessões semanais de privação de sono paradoxal por 48 horas, durante 8 semanas. Esses animais tiveram, após o desmame, a ração padrão substituída por dieta hipercalórica;

5. Grupo Monoglutamato de Sódio (MSG, $\mathrm{n}=6)$ : durante o período neonatal $\left(1^{\circ}\right.$ ao $11^{\circ}$ dia) receberam $2 \mathrm{mg} / \mathrm{kg}$ de peso de monoglutamato sódico, subcutâneo. Esses animais foram utilizados quando completaram três meses de vida e receberam dieta padrão;

6. Grupo Privação de Monoglutamato de Sódio + Sono Paradoxal (MSG+PSP, $\mathrm{n}=6$ ): durante o período neonatal $\left(1^{\circ}\right.$ ao $\left.11^{\circ} \mathrm{dia}\right)$ receberam $2 \mathrm{mg} / \mathrm{kg}$ de peso de monoglutamato sódico. Foram submetidos a sessões semanais de privação de sono paradoxal por 48 horas.

\section{Confecção da dieta hipercalórica}

A dieta hipercalórica que foi produzida no Laboratório de Farmacologia da FCMS/PUC-SP é composta de ração padrão moída, gordura vegetal e leite condensado.

Todos os ingredientes foram homogeneizados e feita uma pasta. Novos pellets foram feitos e deixados secar em estufa de secagem $\left(100^{\circ} \mathrm{C}\right)$ por 12 horas. A dieta hipercalórica foi oferecida ad libidum por 8 semanas.

\section{Privação de sono paradoxal}

Os camundongos foram colocados em uma caixa para ratos que contém seis plataformas de cimento, moldadas em copos pequenos de café. A caixa é preenchida com água até deixar aproximadamente $0,5 \mathrm{~cm}$ da plataforma para fora da água. Durante a sessão semanal de privação (48 horas), os animais receberam água e ração (padrão ou hipercalórica) ad libidum. Camundongos controles permaneceram em suas caixas moradia.

\section{Parâmetros avaliados}

1. Peso corporal: o peso corporal dos animais foi avaliado semanalmente, sempre no mesmo dia da semana e no mesmo período do dia. Para tanto, foi utilizada uma balança semianalítica da marca Marte.

2. Medidas da intolerância à glicose:

- Medida do teste de tolerância oral à glicose: após 12 horas de jejum sólido, uma medida basal de glicemia foi colhida da cauda do animal (a glicemia foi determinada utilizando-se o glicosímetro One Touch). Após as medidas da glicemia basal, $2 \mathrm{mg} / \mathrm{kg}$ de glicose foram administrados por gavagem. Novas amostras de glicemia foram colhidas 30, 60 e 120 minutos após a administração de glicose. A área sob a curva de glicose foi determinada por meio da regra trapezoidal. O cálculo das áreas sob as curvas foi realizado utilizando-se o software GraphPad Prisma;

- $\quad$ Teste de tolerância à insulina: o teste foi realizado após jejum sólido de 6 horas; a primeira coleta de sangue (jejum) foi realizada no tempo basal. Em seguida, a insulina (2 U/kg de peso corporal) foi injetada no peritônio dos camundongos e amostras de sangue foram coletadas pela cauda nos tempos $5,10,15,20,25$ e 30 minutos para a determinação da glicemia. A área sob a curva de glicose foi determinada por meio da regra trapezoidal. O cálculo das áreas sob as curvas foi realizado utilizando-se o software GraphPad Prisma.

3. Medidas de peso ventricular esquerdo, gordura visceral e subcutânea: ao final do teste de tolerância oral à glicose, os animais foram sacrificados, por excesso de anestésico, e o ventrículo esquerdo e a gordura epididimal foram pesadas em balança analítica Marte.

\section{Análise dos resultados}

Os dados são apresentados na forma de média aritmética土erro padrão da média. As variáveis obtidas foram analisadas por métodos estatísticos levando-se em consideração a natureza das variáveis ou a variabilidade das medidas realizadas. A análise da variação do peso corporal, das áreas sob as curvas de glicose durante os testes de tolerância à glicose e à insulina, do peso ventricular esquerdo e da gordura visceral foi feita mediante análise de variância de Mann-Whitney. Foi fixado em 5\% (p<0,05) o nível para a rejeição da hipótese de nulidade.

\section{RESULTADOS}

\section{Variação do peso corporal}

A variação percentual do peso corporal demonstra o ganho percentual de peso entre a oitava semana de estudo e o período basal. Verificou-se que os animais dos grupos DHC e MSG tiveram aumento significante da variação do peso corporal em relação ao CONT. As sessões semanais de privação de sono (grupo PSP) determinaram diminuição significante da variação do peso corporal em relação ao controle. A indução de obesidade atenuou significativamente a diminuição da variação do peso corporal nos grupos DHC+PSP e MSG+PSP. Os dados da variação do peso corporal encontram-se na Figura 1.

\section{Peso da gordura abdominal}

À semelhança do que aconteceu com o peso corporal, a gordura visceral aumentou de maneira significante nos grupos em que houve a produção de obesidade (DHC e MSG). A privação de sono determinou uma diminuição significante da gordura visceral nos três grupos em que foi realizada (PSP, DHC+PSP e MSG+PSP).

\section{Teste de tolerância à glicose e à insulina}

$\mathrm{Na}$ Tabela 1 verificam-se os valores das áreas sob as curvas de glicose durante os testes de tolerância oral à glicose (intolerância à glicose) e a administração intraperitoneal de insulina (resistência à insulina). Verifica-se que, em relação aos animais controles, a indução de obesidade 
(DHC ou MSG) e a privação de sono determinaram um aumento significante da área sob a curva de glicose durante o teste de tolerância oral à glicose. A obesidade induzida por MSG levou a uma maior intolerância à glicose, quando comparada aos animais que receberam dieta hipercalórica; e a sobreposição da obesidade e privação de sono paradoxal não determinaram aumento adicional nesse parâmetro, o que indica que não houve efeito sinérgico entre esses dois parâmetros.

O teste de tolerância à insulina reflete o grau da resistência periférica a esse hormônio. Semelhantemente aos dados obtidos pelo teste de tolerância oral à glicose, os animais submetidos a intervenções (obesidade e privação de sono)

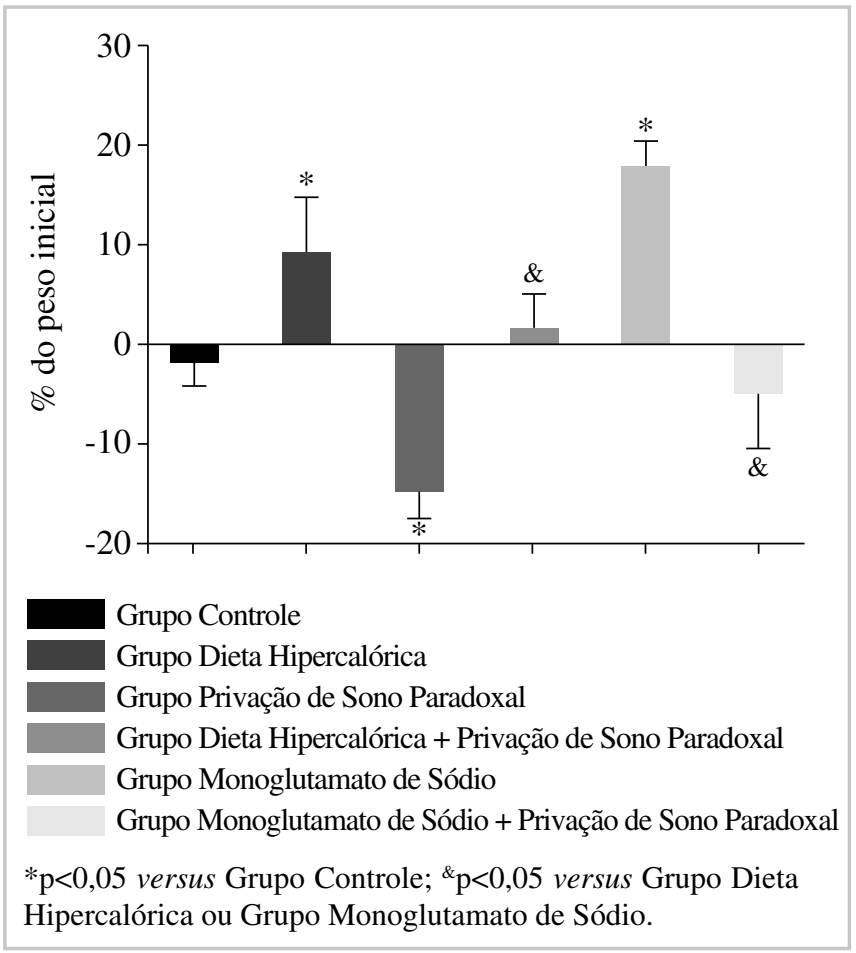

Figura 1. Médiaterro padrão da média da variação do peso corporal (peso na oitava semana/semana basal), medido em porcentagem. tiveram aumento significante desse parâmetro em relação ao controle. Mais uma vez, os animais do grupo MSG e MS$\mathrm{G}+\mathrm{PSP}$ atingiram valores significativamente maiores em relação aos grupos DHC e DHC+PSP. Esses dados estão sumarizados na Tabela 1.

\section{Peso cardíaco relativo}

Nem a indução de obesidade nem a privação de sono dos animais determinaram alterações no peso cardíaco relativo dos animais. Esses dados estão na Tabela 1.

\section{DISCUSSÃO}

Neste trabalho verificou-se que tanto a indução de obesidade quanto a privação de sono paradoxal determinaram piora do metabolismo glicídico dos animais, sendo que nos que receberam dieta hipercalórica houve aumento da gordura visceral. Nos animais PSP, a piora do metabolismo glicídico não se associou ao peso corporal e à gordura visceral. Verificou-se que não houve efeito sinérgico entre PSP e obesidade. O peso cardíaco dos animais não se alterou significativamente.

Neste estudo foram utilizados dois modelos experimentais de obesidade, um modelo exócrino (dieta hipercalórica) e um modelo neuroendócrino (administração neonatal de monoglutamato de sódio, com um modelo de estresse, a privação de sono paradoxal). Obesidade e estresse são dois problemas da sociedade moderna. Existem evidências da literatura que a associação desses dois estímulos pode determinar uma intolerância de glicose e futuramente levar a um quadro de diabetes mellitus tipo $2 .{ }^{21}$ Os modelos de obesidade já estão bem estabelecidos 22,23 e o modelo experimental de privação de sono paradoxal é semelhante ao que poderia acontecer em pacientes que têm uma má qualidade de sono, também com aqueles que têm apneia obstrutiva do sono..$^{15}$

Na fisiopatologia da intolerância à glicose e do diabetes tipo 2 existem vários fatores associados:

- maior ativação simpática e maior secreção de catecolaminas;

- ativação do eixo hipotálamo-hipófise e aumento da secreção de cortisol;

- aumento do estresse oxidativo e aumento da produção de espécies reativas de oxigênio;

Tabela 1. Áreas sob as curvas de glicose nos testes de tolerância oral à glicose e administração intraperitoneal de insulina.

\begin{tabular}{|c|c|c|c|c|c|c|}
\hline & $\begin{array}{c}\text { Grupo } \\
\text { Controle }\end{array}$ & $\begin{array}{c}\text { Grupo Privação } \\
\text { de Sono } \\
\text { Paradoxal }\end{array}$ & $\begin{array}{l}\text { Grupo Dieta } \\
\text { Hipercalórica }\end{array}$ & $\begin{array}{l}\text { Grupo Dieta Hiper- } \\
\text { calórica + Privação } \\
\text { de Sono Paradoxal }\end{array}$ & $\begin{array}{l}\text { Grupo Monoglu- } \\
\text { tamato de Sódio }\end{array}$ & $\begin{array}{c}\text { Grupo Monoglu- } \\
\text { tamato de Sódio + } \\
\text { Privação de Sono } \\
\text { Paradoxal }\end{array}$ \\
\hline $\begin{array}{l}\text { TTGO (mg/ } \\
\text { dL) }\end{array}$ & $10838 \pm 1556$ & $18662 \pm 2302 *$ & $19400 \pm 1857 *$ & $15366 \pm 717^{*}$ & $23723 \pm 3801$ *\# & $22106 \pm 2106^{* \#}$ \\
\hline TTI (mg/dL) & $6510 \pm 572$ & $9582 \pm 1344^{*}$ & $12140 \pm 1552 *$ & $10127 \pm 357^{*}$ & $16898 \pm 1259$ *\# & $15977 \pm 973^{* \#}$ \\
\hline $\begin{array}{l}\text { Peso cardía- } \\
\text { co }(\mathrm{g})\end{array}$ & $0,24 \pm 0,01$ & $0,20 \pm 0,01$ & $0,19 \pm 0,01$ & $0,19 \pm 0,01$ & $0,16 \pm 0,01$ & $0,17 \pm 0,01$ \\
\hline
\end{tabular}

* $\mathrm{p}<0,05$ versus Grupo Controle; " $\mathrm{p}<0,05$ versus Grupo Dieta Hipercalórica ou Grupo Dieta Hipercalórica + Privação de Sono Paradoxal; TTGO: teste de tolerância oral à glicose; TTI: teste de sensibilidade à insulina; PC: peso cardíaco. 
- $\quad$ ativação de vias de inflamação e aumento da secreção de interleucina 6 e TNF- $\alpha$ lfa, bem como

- $\quad$ alteração nas adipocinas com aumento da leptina e diminuição da adiponectina. ${ }^{24}$

Van Cauter et al. ${ }^{25}$ descrevem que a presença de ondas lentas de sono, o sono mais reparador, está associada à diminuição da frequência cardíaca, pressão arterial, atividade nervosa simpática e utilização da glicose cerebral, em comparação com o estado de vigília. Durante o aparecimento dessas ondas, são liberados hormônios anabólicos, como o hormônio do crescimento (GH), enquanto outros hormônios, como o cortisol, são inibidos. Nos últimos anos, evidências laboratoriais e epidemiológicas têm convergido para indicar que a perda de sono pode ser um novo fator de risco para obesidade e diabetes tipo 2. Estudos da literatura verificam, em pacientes com apneia do sono, maiores concentrações de grelina e menores concentrações de leptina, sem, no entanto, associar ao índice de massa corporal (IMC), o que levou os autores a levantar a hipótese de que tais alterações hormonais podem levar ao aumento do risco de obesidade. ${ }^{26}$

É importante destacar, porém, que o aparecimento de distúrbios metabólicos está muito mais associado à qualidade do sono do que ao número de horas dormidas, uma vez que não se encontrou uma correlação entre pacientes insones e distúrbios metabólicos. ${ }^{27}$

Neste trabalho, a piora no quadro metabólico é evidente, com o aumento das áreas sob as curvas de glicose durante os testes de tolerância oral à glicose e o de tolerância à insulina, seja nos grupos tornados obesos seja nos grupos PSP. Porém, em nenhum momento se verificou um efeito sinérgico entre os grupos obesos e os de privação de sono paradoxal. Acredita-se que os mecanismos que levaram à intolerância à glicose sejam os mesmos, mas a ausência de efeito da privação de sono paradoxal sobre o conteúdo de gordura visceral pode ter impedido a piora do quadro metabólico. Em modelo de sono fragmentado e outro de apneia do sono, Wang et al. ${ }^{28}$ encontraram aumento do peso corporal, aumento do consumo alimentar secundário à resistência à leptina e aumento da gordura visceral. Dados semelhantes também são encontrados em estudos clínicos. ${ }^{4}$

O efeito deletério sobre o metabolismo pode ser justificado a partir do modelo experimental, afinal, é sabido que o modelo de dieta hipercalórica, também conhecida como dieta de cafeteria, aumenta a massa de gordura visceral e a atividade simpática dos animais, ${ }^{29}$ induz hiperinsulinemia e hiperleptinemia e determina aumento dos marcadores de inflamação e de radicais livres. ${ }^{30}$ Nos animais MSG, sabe-se que os níveis aumentados de cortisol decorrentes da lesão hipotalâmica, assim como da leptina, decorrentes do acúmulo de gordura visceral e da insulina, consequente à resistência à insulina, podem determinar aumento da atividade simpática, com maior liberação de catecolaminas na terminação nervosa, facilitando o efluxo simpático central. ${ }^{23}$

\section{CONCLUSÃO}

Em conclusão, a administração de uma dieta hipercalórica e a privação de sono paradoxal determinam intolerância à glicose e resistência à insulina, que neste trabalho não foi sinérgico.

\section{REFERÊNCIAS}

1. Spiegel K, Tasali E, Penev P, Van Cauter E. Brief communication: sleep curtailment in healthy young men is associated with decreased leptin levels, elevated ghrelin levels, and increased hunger and appetite. Ann Intern Med. 2004;141(11):846-50.

2. Boergers J, Gable CJ, Owens JA. Later school start time is associated with improved sleep and daytime functioning in adolescents. J Dev Behav Pediatr. 2014;35(1):11-7.

3. Scott LD, Hwang W-T, Rogers AE, Nysse T, Dean GE, Dinges DF. The relationship between nurse work schedules, sleep duration, and drowsy driving. Sleep. 2007;30(12):1801-7.

4. Ayas NT, White DP, Manson JE, Stampfer MJ, Speizer FE, Malhotra A, et al. A prospective study of sleep duration and coronary heart disease in women. Arch Intern Med. 2003;163(2):205-9.

5. Reutrakul S, Van Cauter E. Interactions between sleep, circadian function, and glucose metabolism: implications for risk and severity of diabetes. Ann N York Acad Sci. 2014;1311:151-73.

6. Misra A, Khurana L. Obesity and the metabolic syndrome in developing countries. J Clin Endocrinol Metab. 2008;93(11 Suppl. 1):S9-30.

7. Muñoz-Pareja M, Loch MR, Santos HG, Bortoletto MS, Durán González A, Andrade SM. Factores asociados a mala calidad de sueño en población brasilera a partir de los 40 años de edad: estudio VIGICARDIO. Gac Sanit. 2016;30(6):444-50.

8. Pillar G, Shehadeh N. Abdominal fat and sleep apnea: the chicken or the egg? Diabetes Care. 2008;31(Suppl. 2):S303-9.

9. Morgenstern M, Wang J, Beatty N, Batemarco $\mathrm{T}$, Sica AL, Greenberg H. Obstructive sleep apnea: an unexpected cause of insulin resistance and diabetes. Endocrinol Metab Clin North Am. 2014;43(1):187-204.

10. Cesaretti MLR. Modelos experimentais de resistência à insulina e obesidade: lições aprendidas. Endocrinol Metab. 2006;50:190-7.

11. Colditz GA, Philpott SE, Hankinson SE. The impact of the nurses' health study on population health: prevention, translation, and control. Am J Public Health. 2016;106(9):1540-5.

12. Nilsson PM, Rööst M, Engström G, Hedblad B, Berglund $G$. Incidence of diabetes in middle-aged men is related to sleep disturbances. Diabetes Care. 2004;27(10):2464-9.

13. Meisinger C, Heier M, Loewel H, MONICA/KORA Augsburg Cohort Study. Sleep disturbance as a predictor of type 2 diabetes mellitus in men and women from the general population. Diabetologia. 2005;48(2):235-41.

14. Knutson KL. Impact of sleep and sleep loss on glucose homeostasis and appetite regulation. Sleep Med Clin. 2007;2(2):187-97. 
15. González-Ortiz M, Martínez-Abundis E. Impact of sleep deprivation on insulin secretion, insulin sensitivity, and other hormonal regulations. Metab Syndr Relat Dis. 2005;3(1):3-7.

16. Valensi P, Pariès J, Attali JR, French Group for Research and Study of Diabetic Neuropathy. Cardiac autonomic neuropathy in diabetic patients: influence of diabetes duration, obesity, and microangiopathic complications - the French multicenter study. Metab Clin Exp. 2003;52(7):815-20.

17. Gudbjörnsdottir S, Friberg P, Elam M, Attvall S, Lönnroth P, Wallin BG. The effect of metformin and insulin on sympathetic nerve activity, norepinephrine spillover and blood pressure in obese, insulin resistant, normoglycemic, hypertensive men. Blood Press. 1994;3(6):394-403.

18. Spiegel K, Leproult R, Van Cauter E. Impact of sleep debt on metabolic and endocrine function. Lancet. 1999;354(9188):1435-9.

19. Woods SC, D'Alessio DA. Central control of body weight and appetite. J Clin Endocrinol Metab. 2008;93(11 Suppl. 1):S37-50.

20. Koban M, Swinson KL. Chronic REM-sleep deprivation of rats elevates metabolic rate and increases UCP1 gene expression in brown adipose tissue. Am J Physiol Endocrinol Metab. 2005;289(1):E68-74.

21. van Dijk G, van Heijningen $S$, Reijne AC, Nyakas C, van der Zee EA, Eisel ULM. Integrative neurobiology of metabolic diseases, neuroinflammation, and neurodegeneration. Front Neurosci. 2015;9:173.

22. Ferreira CBND, Cesaretti MLR, Ginoza M, Kohlmann O. Metformin effects upon blood pressure and glucose metabolism of monossodium glutamate induced-obese spontaneously hypertensive rats. Arq Bras Endocrinol Metab. 2009;53:409-15.

23. Voltera AF, Cesaretti MLR, Ginoza M, Kohlmann O. Effects of neuroendocrine obesity induction on systemic hemodynamics and left ventricular function of normotensive rats. Arq Bras Endocrinol Metab. 2008;52:47-54.

24. Briançon-Marjollet A, Weiszenstein M, Henri M, Thomas A, Godin-Ribuot D, Polak J. The impact of sleep disorders on glucose metabolism: endocrine and molecular mechanisms. Diabetol Metab Syndr. 2015;7:25.

25. Van Cauter E, Spiegel K, Tasali E, Leproult R. Metabolic consequences of sleep and sleep loss. Sleep Med. 2008;9(Suppl. 1):S23-8.

26. Verma S, Hussain ME. Obesity and diabetes: an update. Diabetes Metab Syndr. 2016;11(1):73-9.

27. Crönlein T. Insomnia and obesity. Curr Opin Psychiatry. 2016;29(6):409-12.

28. Wang Y, Carreras A, Lee S, Hakim F, Zhang SX, Nair $\mathrm{D}$, et al. Chronic sleep fragmentation promotes obesity in young adult mice. Obesity. 2014;22(3):758-62.

29. Muntzel MS, Al-Naimi OAS, Barclay A, Ajasin D. Cafeteria diet increases fat mass and chronically elevates lumbar sympathetic nerve activity in rats. Hypertension. 2012;60(6):1498-502.

30. Zeeni N, Dagher-Hamalian C, Dimassi H, Faour WH. Cafeteria diet-fed mice is a pertinent model of obesityinduced organ damage: a potential role of inflammation. Inflamm Res. 2015;64(7):501-12. 